\title{
LA PROPUESTA DEL SUMÁK KAWSAY/BUEN VIVIR, EN LOS ESTADOS PlURINACIONALES DE BOLIVIA Y ECUADOR ${ }^{(*)}$
}

\author{
THE PROPOSAL OF THE SUMÁK KAWSAY/BUEN VIVIR, \\ in tHE PLURINATIONAL STATES OF BOLIVIA AND ECUADOR
}

\author{
Vivian Grace Fernández-Dávila Urquidi ${ }^{(* *)}$ \\ Bruna Muriel Huertas Fuscaldo ${ }^{(* * *)}$
}

\begin{abstract}
Resumen: El fortalecimiento de los movimientos sociales indígenas, el nuevo constitucionalismo latinoamericano y los avances de los derechos indígenas en el sistema jurídico internacional son evidencia de las profundas transformaciones políticas y epistemológicas que están ocurriendo en la sociedad contemporánea. Este proceso de transformación culminó en la promulgación de las nuevas constituciones de los Estados Plurinacionales de Bolivia (2009) y Ecuador (2008), que incorporan el principio del Sumák Kawsay/Buen Vivir como propuesta alternativa al modelo de desarrollo hegemónico. El artículo aborda los avances de tal propuesta, desde la perspectiva del proyecto de descolonización política y social de la América Latina.
\end{abstract}

Palabras-Clave: América Latina, Movimientos Indígenas, Estados Plurinacionales, Sumák Kawsay/Buen Vivir.

Abstract: The strengthening of indigenous social movements, the new Latin American Constitutionalism and the advances of indigenous rights at the international legal system demonstrate that deep political and epistemological transformations are taking place in the contemporary society. This process culminated in the promulgation of new constitutions of Bolivia (2009) and Ecuador (2008), that require the Buen Vivir principle, proposed as an alternative to hegemonic development model. The article explores the progress of the propose, from the perspective of the social and political decolonization project for Latin America.

Keywords: Latin America, Indigenous Movements, Plurinational States, Sumák Kawsay/ Buen Vivir.

$(*)$ Este trabajo es el resultado parcial de una investigación de carácter interdisciplinario, realizada a través del Grupo de Investigación Descolonización y Estados Plurinacionales, del Observatorio Interdisciplinar de Políticas Públicas de la Universidad de São Paulo, coordinado por la Dra. Vivian Urquidi y financiado por el Consejo Nacional de Desarrollo Científico y Tecnológico/CNPQ.

$(* *)$ Doctora em Sociología, Profesora de Políticas Públicas y Multiculturalismo en la Escuela de Artes, Ciencias y Humanidades (EACH/USP) y del Programa Interunidades en Integración de la América Latina (PROLAM/USP) de la Universidad de São Paulo, São Paulo, Brasil. E-mail: < vurquidi@usp.br>.

$(* * *)$ Estudiante de Doctorado por el Programa Interunidades en Integración de la América Latina de la Universidad de São

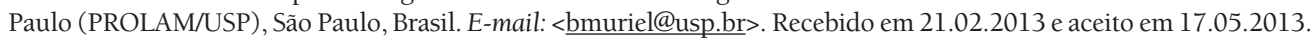




\section{INTRODUCCIÓN}

Los pueblos originarios de América Latina - explotados, subyugados y excluidos desde los principios de la colonización del siglo XV — han sufrido y resistido a acciones que van desde el genocidio hasta los proyectos políticos asimilacioncitas e integracionistas. En las últimas décadas, sin embargo, nuevos procesos políticos y sociales pretenden disminuir las distintas desigualdades y jerarquías (étnico raciales, epistemológicas, político económicas, lingüísticas, entre otras) históricamente construidas. El fortalecimiento de los movimientos sociales indígenas, el nuevo constitucionalismo latinoamericano y los avances de los derechos indígenas en el sistema internacional son evidencias de este proceso, mostrando las transformaciones políticas y epistemológicas de la sociedad contemporánea.

El primer intento de articular los pueblos indígenas en un proyecto nacional, mediante una legislación indígena, se dio en 1940 durante el Primer Congreso Indigenista Interamericano de Pátzcuaro, en México, que desde una perspectiva integracionista preveía el proceso gradual de incorporación de los pueblos indígenas a las comunidades nacionales. Años más tarde, la necesidad de superar el carácter individualista de los derechos humanos en la Declaración de las Naciones Unidas (1948) sería el punto de partida para una serie de acciones que condujeron a la aprobación del Convenio n. 107 sobre los Pueblos Indígenas y Tribales (1957) de la Organización Internacional del Trabajo (URQUIDI, TEIXEIRA, LANA, 2008, p. 2). Aunque se presenten cuestiones como la promoción de los idiomas indígenas, la atribución de tierras tradicionales y el derecho consuetudinario, el objetivo final del Convenio n. 107 era estimular la creación de programas que facilitasen la asimilación de los pueblos indígenas.

Durante la década de 1980 fueron realizadas diversas reformas relacionadas al horizonte pluralista de reconocimiento de los derechos indígenas en ámbitos jurídicos nacionales e internacionales (STAVENHAGEN, 1988; BARIE, 2003). El primer constitucionalismo multicultural fue aprobado en 1982 con la Carta de Derechos y Libertades de la Constitución de Canadá (1982), a lo que siguen las reformas de Guatemala y Nicaragua (1987), en donde se reconocen algunos derechos colectivos y cierto grado de autonomía de los pueblos indígenas. En 1988, la reforma de la constitución brasileña asume la vanguardia regional, garantizando derechos indígenas hasta entonces inéditos. Un año después, algunos de esos derechos fueron incorporados al Convenio 169, sobre pueblos indígenas y tribales, en países independientes, documento de la mayor importancia en defensa de los derechos indígenas dentro del sistema internacional hasta los días de hoy (MARÉS, 1998). En los años noventa del siglo XX, un segundo ciclo de reformas ocurre con la Convención 169 de la OIT, lo que tuvo influencia para promover cambios constitucionales en distintos países de América Latina, entre ellos: Colombia - 1991, México - 1992, Paraguay - 1992, Perú - 1993, Bolivia - 1994, Argentina - 1994, Ecuador - 1996 e 1998 y Venezuela - 1999.

En esta nueva fase de ampliación de los derechos se desarrollaron los conceptos de "nación multiétnica" y "Estado Plurinacional", bien como las fórmulas de pluralismo jurídico. Durante la primera década del siglo XXI la Asamblea General de las Naciones Unidas aprueba la Declaración de las Naciones Unidas sobre el Derecho de los Pueblos Indígenas (2007) y, de manera simultánea, dos procesos constituyentes son realizados, 
visando la construcción de los Estados Plurinacionales: en Bolivia, de 2006 a 2009, en Ecuador, en 2008. Es en ese momento que entra en el escenario el reconocimiento de las formas plurales de organización, producción, selección de las autoridades y de administración de la justicia, además de la promoción de prácticas sociales y valores sintetizados en el principio del Buen Vivir o Sumak Kawsay, como veremos adelante.

Los Estados Plurinacionales andinos afirman, en el plan político, lo que a nivel teórico está siendo discutido en el ámbito del pensamiento social latinoamericano, por lo menos desde José Carlos Mariátegui durante la década de 1920: que la superación de las desigualdades histórico-estructurales de la región depende, en parte, de la capacidad de incorporación de las experiencias milenarias de los pueblos originarios por parte de proyectos nacionales (políticos, económicos, sociales, culturales). Para este autor, el indígena sería el sujeto clave para la construcción de un nuevo proyecto nacional en el Perú que, así como los otros países del continente, sufría las consecuencias "[...] del pecado original de la conquista. El pecado de haber nacido y haberse formado sin el indio y contra el indio" (MARIÁTEGUI, 2007, p. 38). La propuesta de considerar la presencia indígena en los procesos de formación estatal, en los países de América Latina es, por lo tanto, bastante anterior a las reformas pluralistas citadas antes. Pero la intensificación de tal reivindicación y la ampliación de sus conquistas se han dado, principalmente, a partir de la última veintena del siglo XX, amparadas por la acción de distintos actores nacionales e internacionales. Entre ellos, los movimientos sociales indígenas, los intelectuales, las ONGs, los Estados y las Organizaciones Internacionales Gubernamentales.

Además, para que el logro de los avances para los derechos indígenas y, consecuentemente, la ampliación de la capacidad de actuación política de estos pueblos, fue - y es - muy importante la incorporación de categorías y conceptos lo más cerca posible de la realidad de los pueblos indígenas. Se podría citar, por ejemplo, la contribución de Guillermo Bonfil Batalla al debate y su crítica al indigenismo tradicional, aun durante la década de $1970^{(1)}$. El antropólogo mexicano analizó la categoría de "indio" como una concepción eurocéntrica y colonialista, resultado de la dominación efectiva (social, política y económica) sufrida por los pueblos que ya habitaban América Latina antes de la llegada de los europeos.

En el orden colonial el indio es el vencido, el colonizado [...]. De una sola vez, al mismo tiempo, todos los habitantes del mundo americano precolonial entran en la historia europea ocupando un mismo sitio y designados con un mismo término: nace el indio, y su gran madre y comadrona es el dominio colonial [...]. Como toda la estructura colonial, el mundo euroamericano es un mundo escindido, bipolar. El orden jerárquico admite aquí sólo dos instancias: el colonizador y el colonizado. La racionalización correspondiente postula la supremacía del colonizador en base a la superioridad de su raza o de su civilización. (BONFIL BATALLA, 1972, p. 112)

Las nuevas experiencias de Bolivia y Ecuador parecen partir de una postura filosófica y política que percibe al mundo desde la perspectiva de una fundamental igualdad entre

(1) Tanto en su forma de producción teórica como en cuanto proyecto político, el movimiento indígena se basó en un diseño tradicional asimilacionista e integracionista. Su sesgo colonialista se manifiesta en el deseo de superar el retraso y el estado de "pre moderno" de la sociedad en América Latina a través de, entre otras cosas, la asimilación del indígena a la comunidad nacional. 
los pueblos, al mismo tiempo en que prevé la creación de políticas sociales de inclusión, de reducción de las desigualdades y de redistribución de los recursos. Para algunos autores, coincidirían con el proyecto contemporáneo de descolonizar el sistema mundial. Unido a los objetivos de "desmercantilizar" y "democratizar", formarían las claves a través de lo cual deben ser guiadas las actuales prácticas transformadoras contra-hegemónicas (SANTOS, 2007, 2010). Pero ¿qué es exactamente lo que se pretende descolonizar (y desmercantilizar y democratizar)? Un sistema que, en su forma contemporánea, está siendo definido por algunos autores como "sistema-mundo colonial moderno" (QUIJANO \& WALLERSTEIN, 1992).

\section{EL SISTEMA-MUNDO COLONIAL/MODERNO}

El concepto de sistema-mundo colonial/moderno hace referencia a las distintas jerarquías, político-económicas, étnico-raciales, epistemológicas y lingüísticas (entre otras), construidas a partir de la colonización y de la imposición universal del modo de vida capitalista y de los valores occidentales. La primera de ellas, la jerarquía "político-económica", es la que divide el mundo entre centro, semiperiferia y periferia a partir de la división internacional del trabajo y del sistema interestatal de organizaciones político-militares ((WALLERSTEIN, 1979). Complementándola, se observa la jerarquía epistémica, que privilegia la cosmología y conocimientos occidentales frente a los no occidentales (GROSFOGUEL, 2010, MIGNOLO, 2000; QUIJANO, 2005). Juntamente a ello, la "jerarquía lingüística" estructura el privilegio de la comunicación y producción del conocimiento de las lenguas europeas y sus teorías, considerando las otras no como conocimiento/teoría, sino como cultura popular o "folclore" (MIGNOLO, 2000). Por fin, la sociedad estaría organizada a partir de una jerarquía étnico-racial, basada en una estructura de poder específica llamada "matriz de poder colonial" (QUIJANO, 1992, 2005) que, iniciada a partir de los procesos de expansión marítima, se expandió mundialmente y, en especial, con la globalización del siglo XX. En la actualidad, prácticamente todas las dimensiones de la existencia humana (sociabilidad, cultura, mentalidades y subjetividades) son afectadas por esta jerarquía. Así, aunque el colonialismo como fenómeno histórico político de ocupación extranjera, fue dejando de existir en un proceso gradual a partir del siglo XIX, con las luchas por la independencia en América Latina a principios de ese siglo, incluyendo el proceso de descolonización de África y Asia durante las décadas de 1950 y 1960, dentro de la perspectiva de la "colonialidad del poder" (QUIJANO, 1991) seguimos viviendo en un mundo colonizado $^{(2)}$, donde la dominación de clases y la dominación étnico-racial se alimentan mutuamente.

(2) Al final de la década de 1960, Pablo González Casanova y Bonfil Batalla ya trataban del colonialismo interno en América Latina. Estos análisis serán recuperados y reformulados por Aníbal Quijano en la década de 1980. 
La ampliación de los derechos indígenas hasta la formación de los Estados Plurinacionales, por lo tanto, es parte de un proceso que involucra la resistencia a distintos actos de opresión (material, político, moral, intelectual y cultural) que tuvieron inicio, ambos, a partir de la colonización. Con la independencia de los países latinoamericanos, los actos de opresión fueron protagonizados y sostenidos por un Estado que renegó las poblaciones originarias, sus conocimientos, culturas y prácticas sociales. Es en este sentido que la descolonización es un proyecto aún en andamiento, casi dos siglos después de los procesos de independencia.

\section{LOS ESTADOS PLURINACIONALES DE BOLIVIA Y ECUADOR Y SUS NUEVAS CONSTITUCIONES}

Lo que está pasando en Bolivia y Ecuador es parte de un proceso que se intensifica al final del siglo XX, en América Latina, cuando distintos movimientos sociales, incluidos los de base indígena, intensifican sus acciones. En parte, esto correspondió a una reacción al aumento de los problemas sociales causados por los programas de política económica implementados durante la década anterior que, entre otras cosas, orientaban a la privatización, la desregulación y la apertura del mercado, y la no intervención estatal. Distintos países del continente ingresan al nuevo siglo presentando victorias electorales dentro de un proceso que, en América Latina, se identificó como un "giro a la izquierda".

En Bolivia, la victoria de Evo Morales (2005) significó, más allá de la victoria del primer presidente indígena, la transformación de los pueblos originarios en protagonistas de un nuevo proyecto político nacional; de hecho, plurinacional. Así como en Ecuador, con Rafael Correa en la presidencia (2006), la reacción al sistema hegemónico representó un ambicioso proyecto de refundación del Estado a través de las nuevas constituciones que reconocen el pluralismo (político, económico, jurídico, cultural y lingüístico), que existe en el interior de dicho país.

La plurinacionalidad está articulada al concepto de interculturalidad, que predice la diversidad (de naciones) dentro de la unidad (del Estado). Son reconocidas, por las constituciones, deferentes nacionalidades, pueblos indígenas, pueblos afro descendientes y, en el caso de Ecuador, los pueblos montubios (ECUADOR, 2008.) Las constituciones, al afirmar la plurinacionalidad, generaron la necesidad de creación de proyectos nacionales y políticas públicas que sostengan la propuesta plurinacional ${ }^{(3)}$.

El proceso de reordenamiento de los Estados andinos busca incluir, no sin conflictos, de manera estructural la mayoría indígena excluida hasta entonces. Después de la victoria de Morales en 2005, acciones como la obligatoriedad de los idiomas quechua y aymara y la incorporación de los rituales y vestuarios indígenas en eventos oficiales indican el

(3) Es el caso, en Ecuador, donde ha sido creado el Sistema de Educación Intercultural Bilingüe (SEIB), del Ministerio de Educación, que viene implementando en las escuelas públicas de este país la enseñanza del quechua y del shuar. También ha sido creado el sector administrativo de Circunscripciones Territoriales Indígenas, Afro ecuatorianas y Montubias (CTI) para el estímulo de gobiernos territoriales autónomos, con autonomía política, administrativa y financiera. 
intento de transformación de la centenaria institución moderna del Estado, que pasa a considerar la población como parte de la nación. En este sentido, el gobierno propuso la convocación de una nueva Constituyente en que el multiculturalismo boliviano sea incorporado (incluyendo sus formas locales de organización, justicia, la autonomía sobre el territorio y los recursos naturales y la elevación de sus símbolos, idiomas y sistemas míticos) a la categoría de cultura nacional, al lado del español, su bandera y de la religión católica (URQUIDI, 2007, p. 2).

Para algunos pensadores, tales acontecimientos podrían ser indicio de la emergencia de un "debate civilizatorio" inédito: la construcción de una interculturalidad realmente igualitaria que parte de "un encuentro verdaderamente poscolonial" (SANTOS, 2010, p. 60). Serían evidencia de ello los recientes procesos constituyentes de los dos países, que culminaron en documentos sorprendentes para analistas políticos y científicos sociales de todo el mundo. El artículo número 8.1 de la Constitución Política del Estado Plurinacional de Bolivia, por ejemplo, recurriendo a la pluralidad lingüística del país, afirma que "El estado asume y promueve como principios ético-morales de la sociedad plural: (...) suma qamaña (vivir bien), dereko (vida armoniosa), teko kavi (vida buena), ivi maraei (tierra sin mal) y qhapaj ñan (camino o vida noble)" (BOLIVIA, 2009, p. 2). Lo mismo pasó con la Constitución Ecuatoriana de 2008, primera en reconocer la naturaleza como sujeto de derecho ${ }^{(4)}$ y el paradigma del Buen Vivir o Sumac Kawsay (reivindicados por los movimientos indígenas) como condiciones básicas para la refundación de un país étnicamente justo. En el preámbulo de la Constitución, se expresa que son reconocidas las raíces milenarias de un país formado por distintos pueblos:

Celebrando a la naturaleza, la Pacha Mama, de la que somos parte y que es vital para nuestra existencia [...], Como herederos de las luchas sociales de liberación frente a todas las formas de dominación y colonialismo [...] Decidimos construir: Una nueva forma de convivencia ciudadana, en diversidad y armonía con la naturaleza, para alcanzar el buen vivir, el Sumac kawsay (EQUADOR, 2008, p. 1).

Vale la pena retomar la antigua versión de la Constitución de 1830, que nombró “[...] a los venerables curas párrocos por tutores y padres naturales de los indígenas, ejerciendo su ministerio de caridad a favor de esta clase de inocentes, abyecta y miserables" (CHATERS \& STAVENHAGEN, 2009, p. 369). Es decir, los recientes procesos constituyentes en Bolivia y Ecuador parecen cuestionar las centenarias teorías del Derecho y del Estado, al poner en tela de juicio los principios de universalidad e individualidad de las constituciones vigentes hasta entonces, evidenciando una verdadera transición paradigmática en el plano constitucional de estos países (SANTOS, 2010, p. 32). Elevan ciertas experiencias milenarias indígenas al mismo nivel de las prácticas y conocimientos occidentales, afirmando la coexistencia de categorías que provienen de dos universos civilizatorios distintos: Recursos Naturales/Pachamama; Estado-Nación/Estado Plurinacional; Ciudadanía/Derechos Colectivos (SANTOS, 2010). Por fin, Desarrollo/Sumac Kawsay (buen vivir).

(4) Se supone que la naturaleza, o Pachamama, donde la vida es producida y realizada, tiene derecho a que se respete integralmente su existencia y la manutención y regeneración de sus ciclos vitales, además de la restauración, en los casos de impactos ambientales causados por la explotación de los recursos naturales no renovables. (ECUADOR, 2008). 


\section{EL BUEN VIVIR/SUMÁK KAWSAY: UNA ALTERNATIVA EN CONSTRUCCIÓN}

Sumac Kawsay, traducido para el español como Buen Vivir, son los términos más difundidos de un movimiento rumbo a una existencia social diferente de la que predomina hoy ${ }^{(5)}$. Principio de naturaleza ética y política, los términos están presentes a lo largo de los textos constitucionales, lo que manifiesta una valorización positiva de las sociedades indígenas, vistas como sociedades que a lo largo de su proceso histórico han establecido relaciones en armonía con la naturaleza a través de sus acciones comunitarias. La lógica que rige la incorporación del principio por las nuevas constituciones es la de que ciertos fundamentos indígenas podrían ser recuperados para la construcción de una sociedad igualitaria, justa y basada en una concepción de desarrollo distinta de la dominante. El Sumak Kawsay aparece como una alternativa - en términos de concepción, de conocimiento, pero también en términos de prácticas sociales latentes - a una concepción de desarrollo basada en una visión utilitarista de la naturaleza, considerada un objeto externo, inerte e inferior, que debe ser transformada por el conocimiento técnico-científico del ser humano, superior y dominador (SANTOS, 1988).

Aunque la idea de desarrollo lleva siempre subyacente la necesidad de un proyecto social (FURTADO, 1974), el modelo dominante prima por el crecimiento económico, por la industrialización y por la ampliación del mercado consumidor (VEIGA, 2005). Se basa en la universalización de un modelo practicado por los países que han protagonizado la revolución industrial, donde se cristalizó la figura humana como actor transformador del mundo. De tal transformación depende el progreso material y, en consecuencia, el desarrollo social de la humanidad.

[...] uno de los elementos fundamentales de la colonialidad/modernidad/euro centralidad es el nuevo y radical dualismo cartesiano, que separa la razón y la naturaleza. De allí, una de las ideas/imágenes más características del Eurocentrismo, en cualquiera de sus vertientes: la explotación de la naturaleza como algo que no requiere justificación alguna y que se expresa cabalmente en la ética productivista engendrada junto con la revolución industrial (QUIJANO, 2011, p. 12).

En oposición a tal modelo, la mayor parte de las cosmovisiones indígenas sustenta una visión holística de mundo, donde: "[...] se reconoce la condición del hombre como parte del orden cósmico y se aspira a una integración permanente, que sólo se logra mediante una relación armónica con el resto de la naturaleza" (BONFIL BATALLA, 1984, p. 56).

Incorporada por el discurso político de los movimientos sociales indígenas, e incluida en los textos constitucionales de los dos países, la noción de Buen Vivir se relaciona a las formas ancestrales de convivencia de los pueblos indígenas y a la relación de éstos con la naturaleza ${ }^{(6)}$. Pero, más allá de la visión holística de mundo, está relacionada a otros

(5) Otros términos pueden definir el mismo proyecto, dependiendo de la variación del quechua y del Aymara utilizado. Así, Sumac Kawsay, traducido como Buen Vivir, proviene del quechua del sur de Bolivia, mientras Sumac Qamaña proviene del Aymara y es, normalmente, traducido como Vivir Bien. Sumac, entretanto, también significa bello, hermoso, en el norte de Perú y Ecuador. Ya en el norte de Perú y Ecuador, corresponde al término Allin Kg há way (mejor traducido como Buena Manera de Vivir) (QUIJANO, 2011; CELIBERTI, 2010).

(6) Críticos a los procesos boliviano y ecuatoriano están calificando como "corrientes pachamámicas" o "discursos pachamámicos" aquellas teorías y discursos políticos que hacen referencia a las cosmovisiones indígenas como fundamento 
elementos presentes en las prácticas y saberes indígenas que también son contrapuestos a la lógica hegemónica, como las nociones de comunitario, solidaridad y reciprocidad. De modo que, además de proponer otra relación entre ser humano y naturaleza, se configura como una propuesta alternativa al modo capitalista de producción, distribución y consumo: "Es también una alternativa para el mecanismo de reglamentación social (...) una forma de devolver a la sociedad el control sobre la producción" (DÁVALOS, 2010, p. 5).

$\mathrm{Si}$, por un lado, la presencia del Buen Vivir en los textos Constitucionales no significa un cambio abrupto en los sistemas políticos y económicos de esos países, por otro lado se constata que un discurso ha tomado posición. Este deberá ser sostenido por la praxis política de los distintos actores sociales, en especial los movimientos indígenas y por la capacidad de participación en los procesos decisorios de los nuevos gobiernos. La posibilidad del Buen Vivir, como alternativa al concepto de desarrollo dominante, pasa por la desconstrucción de tales conceptos, así como por el abandono de la idea de "valor" de la naturaleza, que se convierte en parte esencial de la sociedad. Lo que no significaría el fin de los avances técnico-científicos: "Una sociedad puede llegar a ser altamente tecnológica y productiva, integrando la naturaleza en su propia visión interna (...) como fundamento y condición de posibilidad de su existencia en el futuro" (DÁVALOS, 2010, p. 7).

Piensadores críticos a los procesos boliviano y ecuatoriano están calificando como "corrientes pachamámicas" o "discursos pachamámicos" aquellas teorías y discursos políticos que hacen referencia a las cosmovisiones indígenas como fundamento para la transformación del sistema actual. "Buscar en esquemas míticos del pasado (Sumak Kawsay o la Pachamama) soluciones para los problemas actuales (...) no pasa de ser una retro-proyección, que además de impedir las transformaciones tampoco movilizan las inteligencias y reales fuerzas de cambió" (PARGA, 2011, p. 31-50). Sin embargo, es perceptible el potencial emancipatorio del Buen Vivir, que podrían estar indicando la emergencia de un nuevo proyecto y una nueva identidad, "[...] histórica, estructuralmente heterogénea como todas las demás, pero cuyo desarrollo podría producir una nueva existencia social liberada de dominación/explotación/violencia [...]" (QUIJANO, 2011).

El Sumák Kawsay aparecería como una oportunidad para construir colectivamente una nueva forma de organización de la vida, siendo "[...] probablemente, la formulación más antigua, la resistencia 'indígena' contra la colonialidad del poder" (QUIJANO, 2011, p. 02). Otro autor afirma que "el Buen Vivir, más que una declaración constitucional, significa (...) una cosmovisión diferente a la occidental, al surgir de raíces comunitarias no capitalistas, existentes no sólo en el mundo andino y amazónico. (...) nos conmina a disolver el tradicional concepto del progreso en su derivación productivista y del desarrollo en tanto dirección única, sobre todo con su visión mecanicista de crecimiento económico" (ACOSTA, 2011, p. 51).

para la transformación del sistema actual. "Buscar en esquemas míticos del pasado (Sumak Kawsay o la Pachamama) soluciones para los problemas actuales (...) no pasa de ser una retro-proyección, que además de impedir las transformaciones tampoco movilizan las inteligencias y reales fuerzas de cambió." (PARGA, 2011, p. 31-50). Sin embargo, en el presente trabajo se parte de la afirmación del potencial emancipatorio del Buen Vivir, aunque se afirme el riesgo del creciente distanciamiento de los actuales gobiernos de Evo Morales y Rafael Corea en relación a los movimientos sociales indígenas. 


\section{CONSIDERACIONES FINALES}

La formación de los Estados plurinacionales como parte de un proceso histórico complejo, que involucra la articulación de los movimientos sociales indígenas y la ampliación de sus derechos en los sistemas jurídicos nacionales e internacionales, parece poner en evidencia nuevas posibilidades para la transformación del sistema-mundo colonial moderno. En este sentido, concordamos con QUIJANO (2011) quién afirma que la propuesta del Sumac Kawsay/Buen Vivir es una cuestión histórica planteada, que exige que sea cuestionada, debatida y practicada.

Desde la conquista, principios propios de la concepción occidental de mundo han sido violentamente impuestos, como el individualismo, el dualismo filosófico, la racionalidad y el antropocentrismo. Valores como la acumulación y la sobrevalorización del derecho a la propiedad privada se impusieron sobre todos los demás derechos, por una visión utilitarista de la naturaleza. Por su vez, marginalizadas desde el inicio del proceso de colonización hasta los días de hoy, las experiencias indígenas han sido descalificadas a lo largo de la historia por el sistema dominante.

Las diferentes instituciones sociales y el pensamiento científico dominante, se esforzaron para producir la "no existencia" de esos conocimientos y prácticas sociales, considerándolos inferiores, improductivos y residuales (SANTOS, 2010). El enfrentamiento de esta herencia histórica depende de la apertura a todo un conjunto de saberes y prácticas que, a lo largo de la historia, han sido producidos por distintos pueblos, contextos y culturas. Boaventura de Souza Santos denomina tales experiencias de "Epistemologías del Sur", donde "sur" en este caso, no corresponde a un concepto geográfico: es antes la metáfora para el sufrimiento causado por el capitalismo y colonialismo en escala global, así como para los procesos de resistencia que fueron emergiendo a lo largo de la historia ${ }^{(7)}$ (SANTOS, 2008).

Las nuevas constituciones plurinacionales podrían, así, evidenciar el diálogo civilizacional necesario a la hora de enfrentar distintos problemas que afectan a la sociedad contemporánea en su conjunto. Es el caso del agotamiento del modelo de desarrollo hegemónico y de la necesidad de construcción de nuevos modelos, basados en una concepción amplia de sustentabilidad. No es un proceso simple y los conflictos se manifiestan. Pero la ampliación de los derechos indígenas en el sistema internacional y la formación de los estados plurinacionales que incorporan categorías indígenas como el Sumak Kawsay, este concepto-proyecto en construcción, demuestran intentos reales hacia a la descolonización de la sociedad y del Estado nuevas perspectivas para el desarrollo de América Latina.

(7) Dos principios fundamentan la idea de "epistemología del sur": por un lado, la "ecología de saberes", el conjunto diverso de conocimientos y experiencias producidas por los pueblos subalternos y tratadas como no existentes por el sistema hegemónico. Por otro lado, la "traducción intercultural", un procedimiento de creación de inteligibilidad recíproca entre las diferentes experiencias de mundo. Este diálogo intercultural solamente es posible partiendo del siguiente presupuesto: todas las culturas son incompletas, luego, el confronto y el diálogo entre ellas posibilitarían un enriquecimiento mutuo. Aumentar la consciencia de la incompletud cultural sería, por lo tanto, una de las tareas previas a la construcción de una concepción multicultural emancipatoria (SANTOS, 2006). 


\section{REFERÊNCIAS BIBLIOGRÁFICAS}

ACOSTA, Alberto. Riesgos y amenazas para el Buen Vivir. Ecuador-Debate 84. Quito: Centro Andino de Acción Popular/CAAP, 2011. p. 51-56.

BOLÍVIA. Constitución política del Estado Plurinacional de Bolívia. 7 de fevereiro de 2009. Disponível em: <http://bolivia.infoleyes.com/shownorm.php?id=469> Acesso em: 05 ago. 2011.

BONFIL BATALLA, Guillermo. El concepto de indio en América. Anales de Antropología. México: Universidad Nacional Autónoma de México, 1972.

México Profundo, una civilización negada. México: Grijalbo, 1984.

BRASIL. Constituição da República Federativa do Brasil. 5 de outubro de 1988. Disponível em: <http:// www.planalto.gov.br/ccivil_03/Constituicao/Constitui\%C3\%A7ao.htm>. Acesso em: 12 ago. 2011.

CASANOVA, Pablo González. Colonialismo interno (una redefinición). Revista Rebeldia, México, n. 12, p. 41-59, out. 2003.

CELIBERTI, Lilian. El Buen Vivir desde la Mirada de las Mujeres. Trabalho apresentado no Congresso Internacional "Las políticas de equidad de gênero en prospectiva: nuevos escenarios, actores y articulaciones". FLACSO- Buenos Aires, Argentina. Nov. 2010. Disponível em: <http://ibase.br/ userimages/ser\%20joven\%20en\%20sudam\%C3\%A9rica.pdf> Acesso em: 03 abr. 2012.

CHARTERS, Claire; STAVENHAGEN, Rodolfo. The UN Declaration on the Rights of Indigenous Peoples: How It Came to be and What it Heralds. In: CHARTERS, Claire; STAVENHAGEN, Rodolfo (eds.). Making the Declaration Work: The United Nations Declaration on the Rights of Indigenous Peoples. Copenhagen, Denmark: International Work Group for Indigenous Affairs - IWGIA, 2009. p. 344-352. Disponível em: <http://www.internationalfunders.org/documents/MakingtheDeclarationWork.pdf> Acesso em: 15 out. 2010.

CLAVERO, Bartolomé. Cultural Supremacy, Domestic Constitutions, and the Declaration of Indigenous People. In: CHARTERS, Claire; STAVENHAGEN, Rodolfo; (eds.). Making the Declaration Work: The United Nations Declaration on the Rights of Indigenous Peoples. Copenhagen, Denmark: International Work Group for Indigenous Affairs - IWGIA, 2009. p. 344-352. Disponível em: <http://www.internationalfunders.org/documents/MakingtheDeclarationWork.pdf> Acesso em: 15 out. 2010.

DÁVALOS, Pablo. Sumac Kawsay: uma forma alternativa de resistência e mobilização. Revista do Instituto Humanitás Unisinos, São Leopoldo, IHU on-line, n. 340, ano X, 2010.

EQUADOR. Constitución del Ecuador. 19 de julho de 2008. Disponível em:<http://www.asambleanacional.gov.ec/documentos/Constitucion-2008.pdf> Acesso em: 1ํㅗ. 2010.

GROSFOGUEL, Ramón. Para descolonizar os estudos de economia política e os estudos pós-coloniais: Transmodernidade, pensamento de fronteira e colonialidade global. In: SOUSA SANTOS, Boaventura de; MENESES, Maria Paula (Orgs). Epistemologia do Sul. São Paulo: Cortez, p. 455491, 2010.

FURTADO, Ceso. O mito do desenvolvimento econômico. Rio de Janeiro: Paz e Terra, 1974.

MARIÁTEGUI, José Carlos. 7 Ensayos de Interpretación de la Realidad Peruana. Caracas: Fundación Biblioteca Ayacucho, 2007.

MIGNOLO, Walter. Historias Locais/Projetos Globais: colonialidade, saberes subalternos e pensamento liminar. Belo Horizonte, Editora da UFMG, 2000.

MORIN, Edgar. Os Sete Saberes necessários à Educação do Futuro. 2. ed. São Paulo: Cortez, 2000.

OIT. Convenção n. 107 da Organização Internacional do Trabalho. Concernente à proteção e integração das populações indígenas e outras populações tribais e semitribais de países independentes, Genebra, em 05 de junho de 1957. Disponível em: <http://www.oas.org/dil/port/1957\%20 
Conven\%C3\%A7\%C3\%A3o\%20sobre\%20Povos\%20Ind\%C3\%ADgenas\%20e\%20Tribais.\%20 (Conven\%C3\%A7\%C3\%A3o\%200IT\%20n\%20\%C2\%BA\%20107).pdf> Acesso em: 05 set. 2011.

. Convenção n.169 da OIT sobre Povos Indígenas e Tribais. Genebra, 1989. Disponível em: <http://www.oit.org.br/sites/default/files/topic/international_labour_standards/pub/convencao\%20169_2011_292.pdf > Acesso em: 02 out. 2011.

ONU. Declaração Universal dos Direitos Humanos. São Francisco, 1945. Disponível em: http://www. un.org/en/documents/udhr/> Acesso em: 04 set. 2011.

Declaração das Nações Unidas sobre os Direitos dos Povos Indígenas. Genebra, 2007. Disponível em: <http://www.un.org/esa/socdev/unpfii/documents/DRIPS_pt.pdf>Acesso em: $1^{\mathrm{o}}$ set. 2011.

PARGA, José Sánchez. Discursos retrorrevolucionários: Sumac Kawsay, derechos de la naturaleza y otros pachamismos. Ecuador-Debate 84.Quito: Centro Andino de Acción Popular/CAAP, 2011, p. 31-50. QUIJANO, Aníbal; WALLERSTEIN, Inmanuel. Americanity as a Concept or the Americas in the Modern World-System. International Journal of Social Sciences, Unesco/ Eres, Paris, n. 134, 1992, pp. 617-627. Disponível em: <http://books.google.com.br/books?id=hNoXnPNS7ngC\&pg=PA10 $4 \& \mathrm{lpg}=\mathrm{PA} 104 \& \mathrm{dq}=\% 22 \mathrm{Americanity}+\mathrm{as}+\mathrm{a}+$ Concept + or + the + Americas + in + the + Modern + Worlds ystem> Acesso em: 15 ago.2011.

Colonialidade do poder, eurocentrismo e América Latina. In: LANDER, Edgardo (Org.). A colonialidade do saber: eurocentrismo e ciências sociais: Perspectivas latino-americanas. Buenos Aires: Colección Sur Sur, CLACSO, 2005, pp. 227-278. Disponível em: <http://bibliotecavirtual.clacso.org.ar/ar/libros/lander/pt/Quijano.rtf> Acesso em: 03 ago.2011.

Quito: CAAP, 2011.

."Buen Vivir" — Entre el desarrollo y la des-colonialidad del poder. Equador Debate,

SANTOS, Boaventura de Souza. Reinvención del Estado y el Estado Plurinacional. Santa Cruz de la Sierrra: SENDA, 2007.

.Conocer desde el Sur. Para una cultura política emancipatoria. La Paz: CLACSO, 2008.

Refundación del Estado en América Latina: Perspectivas desde una epistemología del Sur. Lima: Instituto Internacional de Derecho y Sociedad, 2010.

STAVENHAGEN, Rodolfo. Derecho Indígena y Derechos Humanos en América Latina. México DF: Instituto Interamericano de Derechos Humanos/El Colegio de México, 1988.

Making the Declaration Work. In: CHARTERS, Claire; STAVENHAGEN, Rodolfo (eds.). Making the Declaration Work the United Nations Declaration on the Rights of Indigenous Peoples. Copenhagen, Denmark: International Work Group for Indigenous Affairs - IWGIA, 2009. pp. 352-372. Disponível em: <http://www.internationalfunders.org/documents/MakingtheDeclarationWork.pdf> Acesso em: 15 out. 2010.

URQUIDI, Vivian. A Bolívia do Século XXI: O Impacto do Multiculturalismo e dos Novos Atores Políticos. In: XIII Congresso Brasileiro de Sociologia, 2007, Recife, UFPE. Disponível em: <www. sbsociologia.com.br/portal/index.php?option=com> Acesso em: 08 jul. 2011.

; TEIXEIRA, Vanessa, LANA, Eliana. Questão Indígena na América Latina: Direito Internacional, Novo Constitucionalismo e Organização dos Movimentos Indígenas. Cadernos PROLAM/USP, ano 8, vol.1, p. 199-222, 2008.

VEIGA, José Eli. Desenvolvimento sustentável: o desafio do século XXI. Rio de Janeiro: Ed. Garamond, 2005.

WALLERSTEIN, Immanuel. The Modern World-System. New York: Academic Press, 1979. 